\title{
La Refutabilidad del Sistema de Epiciclos y Deferentes De Ptolomeo
}

\author{
Christián C. Carman \\ Universidad Nacional de Quilmes
}

\begin{abstract}
To assert that the ancient planetary theory proposed by Ptolemy was irrefutableat least until the telescope discovery-is a bit of a cliché. The aim of this paper is to analyze in what sense it could be said that the epicycle and deferent model proposed by Ptolemy to explain the planetary movement is irrefutable and in what sense it is not. To do this, we will use the conceptual framework developed by the Structuralist Conception, and in particular, the Moulines' analysis of the "guiding principles".
\end{abstract}

Keywords: Refutability, ancient astronomy, Ptolemy, guiding principles.

\section{Introducción}

Al menos desde la influyente obra de Thomas Kuhn, ya se acepta que la revolución copernicana no es, como dice él mismo, sólo "una historia de astrónomos y de cielos" ([1957] 1993: 115), sino que aparecen en escena factores no estrictamente científicos que finalmente inclinarían la balanza a favor de Copérnico. Esto ha llevado a creer que la teoría planetaria de Ptolomeo, entendida exclusivamente como un sistema de órbitas circulares y uniformes con la Tierra en el centro, es en sí misma irrefutable (al menos hasta la aparición del telescopio) y que sólo por su inseparable vinculación con aspectos meta-astronómicos entre los que primaron, sin duda, los de la física y cosmología aristotélica, la teoría podría ser refutada. Probablemente, la lectura histórica sea la correcta y el sistema de epiciclos y deferentes finalmente cayó arrastrado por el derrumbe de la cosmología aristotélica; pero a nivel conceptual, claramente puede distinguirse entre el hecho de que haya sido abandonada por cuestiones parcialmente externas a ella, de la atribución de la propiedad de irrefutabilidad. Es claro que la irrefutabilidad no le ha sido atribuida solamente por factores de tipo histórico, sino también, en gran medida, porque la estructura de epiciclos y deferentes parece una maquinaria de adhocidad genialmente pensada que puede hacer frente a los movimientos más extraños (cfr. Hanson 1960).

El objetivo de este trabajo no es de tipo histórico en el sentido de que no pretende analizar la evidencia histórica ni proponer ni discutir ninguna hipótesis histórica respecto de las razones por las cuales la astronomía ptolemaica fue abandonada. Su objetivo es de análisis conceptual: pretende explicitar en qué sentido el sistema de epiciclos y deferentes tal cual fue presentado por Ptolomeo es irrefutable y en qué

Principia 14(2): 211-239 (2010).

Published by NEL — Epistemology and Logic Research Group, Federal University of Santa Catarina (UFSC), Brazil. 
sentido no lo es. La respuesta final no tendrá mucha novedad: será irrefutable si se lo considera independientemente de una física y, en cambio, se volverá refutable en la medida en que se introduzcan tesis físicas, tales como la que postula la no existencia del vacío o que la naturaleza no hace nada en vano. Pero, creemos, el valor del presente estudio consiste en la precisión con la que aquella respuesta general puede expresarse. Utilizaremos para nuestro análisis las herramientas conceptuales provistas por la concepción estructuralista de las teorías por dos razones. En primer lugar porque creemos que ha alcanzado una sutileza difícil de encontrar hoy en el mercado. En segundo porque C. Ulises Moulines analiza dentro de esta concepción la noción de principio guía y le atribuye la propiedad de ser empíricamente irrestricto. En diálogo con esta noción se construye gran parte de nuestro análisis. Sin embargo, evitaremos cualquier formalización (típicas de la concepción estructuralista) para no intimidar lectores no afectos a las fórmulas. Nos hemos tomado el trabajo de explicar brevemente aquellas nociones del estructuralismo de las que haremos uso para que el lector no familiarizado con ellas pueda, sin embargo, comprender lo aquí propuesto.

El trabajo, entonces, se organiza de la siguiente manera: a) una primera parte en la que expondremos brevemente la noción de principio guía propuesta por Moulines; b) una segunda, en la que haremos una presentación intuitiva de los aspectos centrales de una reconstrucción estructuralista del núcleo teórico de la teoría planetaria de Ptolomeo (TPP a partir de ahora), aunque en términos informales; c) una tercera, en la que analizaremos la irrestricción empírica del TPP sin considerar las leyes especiales; d) una cuarta, en la que describiremos brevemente las leyes especiales más relevantes, algunas condiciones de ligadura y analizaremos cómo afecta a la irrestricción empírica el agregado de las leyes especiales y f) una conclusión. Nuestra investigación intentará aportar claridad y precisión a la cuestión de la irrefutabilidad de la teoría ptolemaica.

\section{Los principios guías según Moulines}

Resumiremos en un párrafo, de manera sumamente esquemática, los conceptos fundamentales de la concepción estructuralista que necesitaremos para nuestro desarrollo. ${ }^{1}$ La unidad mínima de análisis de una teoría científica para la concepción estructuralista es el elemento teórico que posee una parte formal, llamada núcleo teórico y una aplicativa, denominada dominio de aplicaciones pretendidas. Intuitivamente, lo central del núcleo teórico serán las leyes que se aplicarán a sistemas empíricos reunidos en las aplicaciones pretendidas. Pero, conforme a la metateoría estructuralista, lo relevante no es la manera lingüística concreta de expresión de una ley o de varias leyes, sino las estructuras modelo-teóricas que satisfacen dicha ley o dichas leyes. El

Principia 14(2): 211-239 (2010). 
núcleo está formado por una serie de modelos: los modelos potenciales que serán las estructuras que satisfacen los axiomas impropios, es decir, aquellos que especifican el tipo lógico de las estructuras. De ellos tiene sentido preguntarse si cumplen o no con la ley de la teoría. Si efectivamente la cumplen, serán modelos actuales. Pero los modelos potenciales no sólo son restringidos por las leyes, también hay condiciones de ligadura que imponen restricciones entre los modelos de una misma teoría y vínculos interteóricos que, relacionando componentes de una teoría con componentes de otra, también imponen restricciones adicionales. Como hemos visto, los modelos potenciales expresan el aparato conceptual de la teoría, pero dentro de ese aparato es conveniente distinguir el propio de esa teoría del que no lo es (distinción T-teórico, T-no teórico). El último componente del núcleo teórico son los modelos potenciales parciales que resultan de "recortar" a los modelos potenciales los componentes teóricos de esa teoría. Por otro lado, una ley fundamental es aquella que debe cumplir todo modelo actual. Pero una teoría tiene, además, leyes especiales, que son aquellas que van especificando, para ciertos modelos potenciales, determinados valores. Éstas no deben ser cumplidas por todos los modelos actuales y permiten ir armando un árbol invertido en el que se clasifican los distintos tipos de leyes especiales y, de esa manera, se van agrupando los distintos tipos de modelos de la teoría. Así, por ejemplo, la ley fundamental de la mecánica clásica es el segundo principio de Newton, pero que luego es especificado con una serie de leyes especiales que definirán distintos tipos de fuerza: la ley de gravitación universal, la ley de la fuerza de rozamiento, de fricción, etc. Las distintas leyes especiales, al ir determinando las variables, vuelven refutable a la teoría que, si consistiera sólo en la ley fundamental, no podría ser refutada.

En Forma y función de los principios guías, Moulines (1978/1982) presenta una fecunda caracterización de lo que él considera un principio guía mediante un análisis del concepto de fuerza introducido por la mecánica clásica. La intuición básica que el análisis intenta rescatar es que 'no todas las peculiaridades del concepto de fuerza quedan suficientemente analizadas al subsumirlo bajo la categoría sneediana de magnitud T-teórica' (p. 95), pues masa y fuerza son términos teóricos pero 'no pueden ponerse, por así decir, al mismo nivel. Si la masa es un concepto T-teórico, como parece serlo, entonces la fuerza, además de T-teórica, es otra cosa; es más abstracta todavía.' (p. 95)

Por un lado, el segundo principio de la mecánica clásica es —en términos de Moulines - empíricamente irrestricto puesto que 'a pesar de que ... no puede tomarse como una definición, su estructura es tal que ... cualquier modelo parcial (no-teórico) puede ser "extendido" o "completado" trivialmente hasta transformarse en un modelo completo (teórico) de la mecánica" (p. 96). Pero, por otro lado, es un principio muy fecundo que orienta con mucho éxito al científico con respecto a lo que debe buscar cuando analiza el movimiento de los cuerpos. Según Mouli- 
nes, 'es precisamente la estructura [lógica] de [fuerza] lo que hace al principio tan fecundo, a pesar de ser empíricamente irrestricto y aunque esto suene a paradoja metodológica' (p. 96).

En efecto, Moulines destaca que, en las reconstrucciones típicas, 'fuerza y masa aparecen ... "al mismo nivel" [pues] ambas son funciones de las partículas, es decir, de las variables primitivas del sistema' (p. 97). Pero 'para explicar la forma lógica del segundo principio hay que cuantificar sobre variables funcionales y no sólo individuales; es decir, si queremos formalizar necesitamos al menos una lógica de segundo orden. La razón no es meramente que debemos introducir distintas funciones fuerza ..., sino que tales funciones fuerza son funciones de funciones ... y no simplemente funciones de partículas e instantes. ... Cada sistema mecánico se describe mediante cierto número de fuerzas y cada una de estas fuerzas es un funcional.' (pp. 97-8)

En efecto, 'para cada sistema físico, las fuerzas consideradas ... dependen de una serie de "parámetros adicionales" que sólo se especifican en el momento de la aplicación concreta' (p. 98). Parámetros que son, a su vez, 'funciones de las variables individuales (básicamente partículas e instantes)' (p. 98), por ejemplo 'coordenadas espaciales, ... , velocidades, masas, cargas eléctricas, polos magnéticos, coeficientes elásticos, coeficientes de fricción, etc. Todos ellos son, naturalmente, funciones de partículas y/o instantes.' (p. 98)

'El aspecto más significativo [del segundo principio] desde un punto de vista metodológico es la gran cantidad de cuantificadores existenciales involucrados en este enunciado' (p. 100). Pero es un hecho bien conocido que 'si el dominio sobre el que discurren los cuantificadores existenciales es potencialmente infinito, entonces ... el enunciado ... es irrefutable empíricamente' (p. 100). Esta irrefutabilidad puede ser considerada de primer orden ya que es debida a la cuantificación sobre variables individuales de un dominio. Pero la irrefutabilidad del segundo principio es una " "irrefutabilidad de segundo orden", dado que se cuantifica existencialmente sobre variables de segundo orden. En realidad, la característica indeterminación del principio no proviene de su cuantificación existencial sobre realidades empíricas (cuerpos, lugares, instantes) sino sobre funciones teóricas y sobre funcionales abstractos. Es decir, se trata aquí de una cuantificación sobre las potencialidades matemáticas de nuestra mente al tratar con problemas empíricos' (p. 101).

\section{Presentación intuitiva estructuralista de la TPP}

Nadie mejor que Ptolomeo para definir cuál es el objetivo de su teoría planetaria. En la introducción al libro que trata el movimiento planetario, afirma:

Ahora, nuestro objetivo es demostrar para los cinco planetas, de la misma manera que lo hemos hecho para el Sol y la Luna, que todas sus aparentes

Principia 14(2): 211-239 (2010). 
anomalías pueden ser representadas por movimientos uniformes y circulares, puesto que ellos son propios de la naturaleza de los seres divinos, mientras que la no-uniformidad y el desorden les son ajenos. Por lo tanto, es correcto que deberíamos pensar que el éxito en un propósito tal es una gran cosa, y ciertamente el fin propio de la parte matemática de la filosofía teórica. (IX, 2; H2-208; T420) ${ }^{2}$

Ptolomeo sostiene, por lo tanto, que el fin propio de la parte matemática de la filosofía teórica es reducir la trayectoria aparente de los astros a movimientos uniformes y circulares. El mecanismo utilizado para ello podía ser o bien las esferas concéntricas de Eudoxo o bien el sistema de epiciclos y deferentes propuesto por Apolonio. Ptolomeo opta por éste último. Por lo tanto, la ley fundamental de la TPP debería decir algo así: para cada planeta existe al menos un sistema de epiciclos y deferentes tal que la posición calculada por ese sistema es igual a la posición observada, en cualquier instante de tiempo.

En primer lugar, entonces, habría que caracterizar los conjuntos base que serán el conjunto de los planetas, el de instantes de tiempo y el de los puntos en el espacio. Luego habría que caracterizar las funciones que corren sobre esos conjuntos. Tendríamos allí una función posición que permita ubicar cualquier punto del espacio mediante tres coordenadas: la latitud celeste, la longitud celeste — ambas expresadas en ángulos (que eran las que utilizaba Ptolomeo para ubicar un planeta sobre el fondo de estrellas fijas) - y una tercera coordenada que nos diera la distancia entre el centro de la Tierra y ese punto. Habrá que agregar una función que atribuya a cada planeta, en cada instante de tiempo, un brillo particular. También es necesario un conjunto de órbitas que podría caracterizarse como un subconjunto teórico del conjunto de los puntos o también introducirse como un conjunto de individuos teóricos. Sea como fuere, el conjunto de órbitas estará partido en dos subconjuntos: el de deferentes y el de epiciclos. ${ }^{3}$ Sobre las órbitas, a su vez, correrán algunas funciones: la primera le atribuye un punto que será el centro de la órbita, la segunda le atribuye un radio y la tercera le atribuye un punto móvil que será el que se desplace con una determinada velocidad angular (que será la cuarta función) con ese centro y ese radio. La velocidad angular medida desde un punto interior a la órbita es constante; a menos que exista un punto ecuante, ese punto coincide con el centro de la órbita. Sobre el punto móvil estará ubicado el centro del planeta (si es el último epiciclo) o el centro de la siguiente órbita (si es un deferente o un epiciclo interior). Que el radio de las órbitas no varíe, hará que sean circulares (porque siempre el punto móvil estará equidistante del centro), y que la velocidad angular no esté en función del tiempo garantizará que sea constante. Que el centro del deferente sea la Tierra es la expresión del geocentrismo. Si queremos que el sistema admita excéntricas debemos permitir que el centro del deferente no sea el centro de la Tierra, pero que no se aleje demasiado para que siga siendo cualitativamente geocéntrico. 
Ahora contamos con todos los elementos para poder describir una órbita. Pero la teoría no propone órbitas aisladas sino un sistema de epiciclos y deferentes (sed a partir de ahora) que es, justamente, un sistema que encastra de una manera particular a varias órbitas. Deberá existir, entonces, una función que vincule órbitas, montándolas unas sobre otras de una manera muy precisa: una órbita $b$ está montada sobre otra órbita a si y sólo si, en todo tiempo, la posición del centro de la órbita $b$ es igual a la posición del punto móvil de la órbita $a$. El conjunto de órbitas montadas unas sobre otras formará una cadena y el punto móvil de la última órbita será el punto móvil de todo el sistema.

La ley fundamental, entonces, diría que para cada planeta existe un sed tal que la longitud y latitud celestes de su punto móvil coincida con la longitud y latitud celestes observadas del planeta y, además, que el brillo del planeta observado sea (cualitativamente) inversamente proporcional ${ }^{4}$ a la distancia Tierra-Planeta en cualquier instante de tiempo. ${ }^{5}$

\section{La irrestricción empírica de la ley fundamental del SED}

Ahora nos preguntaremos si la ley fundamental que hemos propuesto para la TPP puede ser considerada un principio guía o se trata sólo de una ley fundamental, centrándonos, fundamentalmente en si es o no empíricamente irrestricta. Por supuesto, afirmar que la ley fundamental de una teoría, tomada aisladamente, es irrefutable no es lo mismo que afirmar que la teoría en sí lo es, ya que ésta no se reduce a su ley fundamental, aun cuando indudablemente la ley fundamental cumple un papel esencial en la caracterización de la teoría.

Tal como lo hemos presentado, la ley fundamental de la TPP introduce claramente cuantificadores existenciales, pues sostiene que existe un sed tal que la posición de su punto móvil coincide con la observada. Esto sólo basta para atribuirle la facultad de ser irrefutable desde la experiencia. En el caso de la mecánica -como hemos visto- si la sumatoria de fuerzas no coincide con el producto de la masa y la aceleración, uno siempre podía suponer la presencia de fuerzas todavía no medidas. De la misma manera, en el caso de la TPP si no se logra predecir con exactitud la posición observada de un planeta, siempre se puede suponer que no se ha encontrado la combinación adecuada de deferentes y epiciclos.

Pero la experiencia no es la única forma de refutar un enunciado con cuantificadores existenciales, pues el enunciado podría afirmar, por ejemplo, algo analíticamente falso. El enunciado "existe un padre que nunca ha tenido hijos", si bien es irrefutable desde la experiencia, es perfectamente refutable analíticamente. Por otro lado, el enunciado "existe un cuervo blanco" es irrefutable desde la experiencia a causa de su estructura lógica y, como tampoco puede ser refutado analíticamente, es

Principia 14(2): 211-239 (2010). 
simplemente irrefutable o irrefutable simpliciter. Diremos, entonces, que un enunciado es irrefutable simpliciter cuando lo es tanto analítica como empíricamente. Cuando los enunciados existenciales vinculan relaciones matemáticas, la situación es parcialmente diferente. Está claro que "existe un número finito de fuerzas tales que, sumadas, dan por resultado el producto de la masa por la aceleración” es irrefutable empíricamente por el sólo hecho de poseer un cuantificador existencial que corre sobre un dominio infinito. También está claro, en este caso, que no puede ser refutado analíticamente, ya que es fácil ver matemáticamente que, para cualquier valor resultante del producto de la masa por la aceleración, es posible inventar un conjunto finito de fuerzas cuya sumatoria coincida con ese valor. La simplicidad de la ecuación matemática del segundo principio nos permite ver eso de una manera tan inmediata que, por lo general, ni lo tematizamos.

Pero la situación es claramente distinta con la ley fundamental de la TPP. Nuevamente, está claro que es irrefutable empíricamente, pero ¿lo es analíticamente? A simple vista no hay nada en la expresión de la ley que nos asegure que es imposible probar que existe un caso para el que eso no es posible. Si un planeta, por ejemplo, tuviera una trayectoria cuadrada ¿podría encontrarse un conjunto de epiciclos y deferentes que describieran esa órbita? ¿no podría probarse a priori que no se lo encontrará? ¿Y si la trayectoria tuviera la forma de una flecha? ¿Y si fuera tan compleja que reprodujera el rostro de Homero J. Simpson? ¿Quién puede asegurarnos que, incluso en esos casos, podrá encontrarse un sed? Afortunadamente, Jean Baptiste Joseph Fourier puede.

En un artículo injustamente olvidado, Hanson (1960) prueba que es posible interpretar el sistema de epiciclos y deferentes como series de Fourier y, por lo tanto, que existe un sed para cualquier función que se comporte lo suficientemente bien, o sea, para cualquier órbita con tal de que sea continua, acotada y periódica.

En efecto, la intuición fundamental del desarrollo en series de Fourier es que cualquier función, aunque presente una forma sumamente compleja, siempre que se comporte bien (continua, acotada y periódica) puede descomponerse en una suma de funciones sinusoidales relacionadas armónicamente (es decir, que sus períodos sean múltiplos enteros entre sí).

Supongamos que representamos la ubicación de un punto z en el plano complejo. Las ordenadas estarán dadas por la parte imaginaria de este número, mientras que las abscisas por su parte real, donde $i$ es la unidad imaginaria y se define como la raíz cuadrada de -1 .

Si llamamos $\rho$ a la distancia desde $z$ hasta el origen, y $\theta$ al ángulo comprendido entre $z$ y las abscisas, podemos definir a $z$ de la forma:

$$
z=a+b i=\rho \cdot e^{i \cdot \theta}
$$

De esta manera tendremos, para el mismo número z, sus coordenadas cartesiaPrincipia 14(2): 211-239 (2010). 


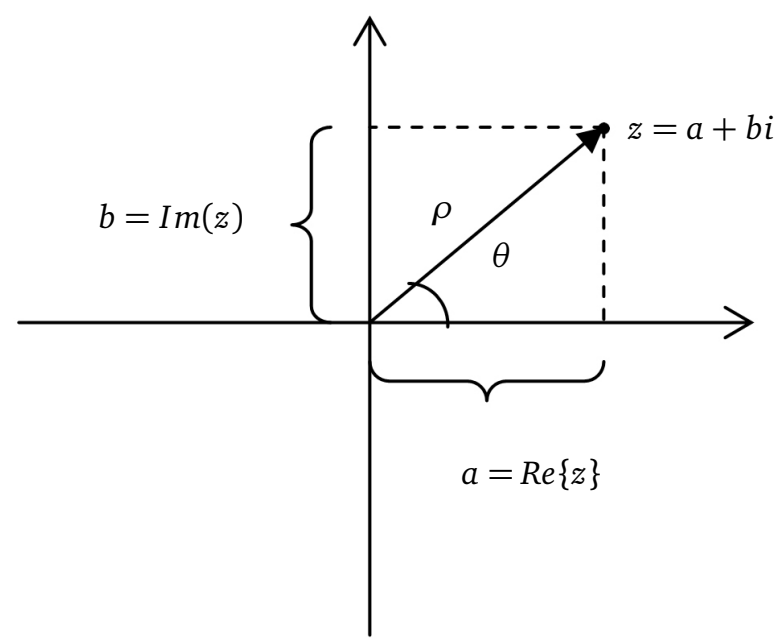

nas $a$ y $b$, y sus coordenadas polares $\rho$ y $\theta$, siendo $\rho$ un número complejo y $\theta$ real.

Si un punto $z_{3}$ está definido por la suma de otros puntos $z_{1}$ y $z_{2}$, entonces la ubicación se encuentra en:

$$
z_{3}=z_{1}+z_{2}=\rho_{1} \cdot e^{i \cdot \theta_{1}}+\rho_{2} \cdot e^{i \cdot \theta_{2}}
$$

Donde $r_{1}$ y $\theta_{1}$ y $r_{2}$ y $\theta_{2}$ son las coordenadas polares de $z_{1} \mathrm{y} z_{2}$, respectivamente.

Un movimiento circular y uniforme, con centro $z_{c}$, radio $r$ y velocidad angular $\omega$ queda representado de la forma:

$$
z=z_{c}+r \cdot e^{i \cdot \omega \cdot t}
$$

Donde $t$ representa al tiempo, ya que el ángulo $\theta$ es igual al producto de la velocidad angular $(\omega)$ y el tiempo $(t)$.

Si el centro es un punto fijo, $z_{c}=r_{c} \cdot e^{i \cdot \theta_{c}}$, tenemos:

$$
z=r_{c} \cdot e^{i \cdot \theta_{c}}+r \cdot e^{i \cdot \omega \cdot t}
$$

Si el centro del movimiento es un punto móvil, definido por una función $f(t)$, entonces:

$$
z=f(t)+r \cdot e^{i \cdot \omega \cdot t}
$$

Como un caso particular, si el centro del movimiento circular y uniforme es, a su vez, otro movimiento circular y uniforme con radio $r^{\prime}$ y velocidad angular $\omega^{\prime}$, obtenemos:

$$
z=r^{\prime} \cdot e^{i \cdot \omega^{\prime} \cdot t}+r \cdot e^{i \cdot \omega \cdot t}
$$

Principia 14(2): 211-239 (2010). 
De esta manera, una suma de movimientos circulares y uniformes quedará expresada de la forma:

$$
z=a_{1} \cdot e^{i \cdot \omega_{1} \cdot t}+a_{2} \cdot e^{i \cdot \omega_{2} \cdot t}+a_{3} \cdot e^{i \cdot \omega_{3} \cdot t}+\ldots+a_{n} \cdot e^{i \cdot \omega_{n} \cdot t}
$$

Donde $a_{1}, a_{2}, \ldots, a_{n}$ son números complejos y representan el radio del movimiento circular y $\omega_{1}, \omega_{2}, \ldots, \omega_{n}$, las velocidades angulares.

Asumimos la trayectoria de un planeta como una función $f(t)$ periódica, acotada y continua:

$$
z=f(t)
$$

Para simplificar, podemos asumir que su período es $2 \pi$, esto es, que $f(t+2 \pi)=$ $f(t)$. Entonces, según el desarrollo en series de Fourier, $f(t)$ se puede representar por una serie

$$
f(t)=\sum_{-\infty}^{+\infty} C_{n} e^{i \cdot n \cdot t}
$$

En esta serie, los coeficientes $\left(C_{n}\right)$ pueden ser considerados como los radios de las órbitas ( $r, r^{\prime}$ en la ecuación 6 y $a_{n}$ en la 7) y $n$ como la velocidad angular (anteriormente $\omega, \omega^{\prime}$ ) y así logramos la misma expresión de la cadena de órbitas de la ecuación 8. Por lo tanto, cada término de esta sumatoria representará una órbita (un epiciclo o el deferente). Se necesitan infinitos términos para lograr que la suma de epiciclos y deferentes copie perfectamente la trayectoria del planeta, pero Fourier predice que siempre vamos a encontrar un conjunto finito de epiciclos que se aproximen a la trayectoria real tanto como se quiera.

Hay que tener en cuenta, sin embargo, que el representar a los epiciclos y deferentes como series de Fourier impone a éstos tres limitaciones que Ptolomeo no tenía por qué respetar. En primer lugar, las velocidades angulares en Fourier ( $n$ ) deben guardar relación armónica, es decir, son múltiplos enteros de la fundamental. En segundo, los coeficientes de la serie $\left(C_{n}\right)$ que representan los radios de los epiciclos, están dados por una fórmula fija:

$$
C_{n}=\frac{1}{2 \pi} \cdot \int_{0}^{2 \pi} f(t) \cdot e^{-i \cdot n \cdot t} d t
$$

Y en tercer lugar las series de Fourier no permiten ni excéntricas ni ecuantes, artificios que Ptolomeo sí utilizaba. ${ }^{6}$ Ahora bien, estas tres restricciones — sobre todo la última - aunque ha llevado a algunos autores a sostener que no es lícito representar al sistema planetario ptolemaico como series de Fourier, lo único que indica, en nuestro contexto, es que Ptolomeo tenía más libertad que Fourier, es decir que si Fourier puede encontrar una solución, también era posible que Ptolomeo, que contaba con menos restricciones, ${ }^{7}$ la encontrara. 
Por lo tanto, si Hanson tiene razón y la prueba de Fourier es válida, entonces la ley fundamental de la TPP no sólo es irrefutable, sino que es verdadera, e.e. válida en toda aplicación intencional, pues garantiza que puede hallarse, para cualquier trayectoria (que se comporte bien), el sistema de epiciclos y deferentes correspondiente.

Hay, por supuesto, restricciones que Ptolomeo imponía al sistema y que en principio no tendrían porqué ser respetadas por Fourier, pero ellas son, justamente, las leyes especiales y las trataremos luego, en la sección siguiente.

Ahora bien, ¿cuál es el valor exacto de la prueba de Fourier? Para responderlo, debemos hacer algunas aclaraciones.

Ya hemos hecho la distinción entre irrefutabilidad empírica e irrefutabilidad analítica, pero hasta ahora hemos considerado prácticamente como sinónimos dos expresiones que utiliza Moulines: la de "irrefutabilidad empírica" y la de "empíricamente irrestricto".

Irrefutable — todos lo sabemos - se predica de una proposición cuya falsedad no puede ser probada. Sabemos, además y como bien lo aclara Moulines, que las proposiciones que poseen cuantificadores existenciales de un dominio potencialmente infinito, son empíricamente irrefutables por su misma estructura lógica. Por supuesto, ello no quiere decir que la proposición sea verdadera (tal cosa sería incurrir en la falacia ad ignorantiam), sino sólo que partiendo de la experiencia no puede probarse su falsedad.

Por otro lado, Moulines introduce la expresión "empíricamente irrestricto" para aquellas leyes cuya estructura garantiza que cualquier modelo potencial parcial puede ser completado trivialmente hasta transformarse en un modelo completo. Es decir, dada la estructura de la ley fundamental, se puede garantizar que será verdadera para todos los modelos potenciales parciales. Cualquier sistema empírico del que tenga sentido predicarse la ley, se la predicará con verdad. Pero el ser empíricamente irrestricto sólo garantiza que se puede completar de una manera trivial.

Un modelo potencial es completado trivialmente cuando lo es puramente ad hoc. Por ejemplo, dada cualquier masa $m_{1}$ y cualquier aceleración $a_{1}$, no es nada difícil obtener una sumatoria de fuerzas que sea igual al producto $m_{1} \cdot a_{1}$. Si el producto fuera igual a 10, los valores de las fuerzas podrían ser 5 y 5 ó 15 y -5 ó 1 y 2 y 7 o infinitas combinaciones posibles. Evidentemente esta forma trivial de completarlo no tiene mucha relevancia para el físico, pues no tiene ningún significado empírico.

La forma de destrivializar la extensión de un modelo potencial a modelo es agregando leyes especiales, condiciones de ligadura y/o vínculos interteóricos que determinen no arbitrariamente al menos algunas de las variables que intervienen en la ley fundamental, de tal manera que su aplicación hace que al menos un modelo potencial no pueda convertirse en modelo. No basta con que determine algunas variables, pues, por ejemplo, una ley especial que sostuviera que la masa y la aceleración nun- 
ca pueden tener valores iguales (por ejemplo, que si la sumatorias de las fuerzas es 4 , la aceleración no puede ser 2 si la masa es 2), restringiría ciertamente los valores que puede tomar la masa, pero, de todas maneras, cualquier modelo potencial podría todavía ser convertido en modelo. Así, no basta con que la ley especial determine algunos valores sino que es necesario que esa determinación excluya algunos modelos potenciales.

Al afirmar que una determinada ley fundamental es (empíricamente o simpliciter) irrefutable y empíricamente irrestricta le estamos atribuyendo, por lo tanto, propiedades distintas. En el primer caso afirmamos que su falsedad no puede ser probada (simpliciter o empíricamente) y, en el segundo, que sus modelos parciales pueden ser trivialmente convertidos en modelos. Está claro que la propiedad de ser empíricamente irrestricto implica la de irrefutabilidad simpliciter (y, por lo tanto, también la empírica) porque, si todo modelo potencial puede ser convertido en modelo, no hay forma de refutar la ley fundamental. Pero la irrefutabilidad empírica no implica necesariamente la propiedad de ser empíricamente irrestricto y esto porque el mero hecho de introducir cuantificadores existenciales que corran bajo un dominio potencialmente infinito no garantiza por sí mismo que cualquier modelo potencial podrá ser convertido en modelo, ni siquiera trivialmente. Frente a un aparente fracaso de la teoría, es decir, frente a un modelo potencial que no se logre convertir en un modelo exitoso, la existencia de cuantificadores existenciales permite afirmar que aún no hemos encontrado la forma de hacerlo (y eso basta para que sea empíricamente irrefutable) pero no permite asegurar que lo encontraremos ni siquiera trivialmente (y eso es necesario para que sea empíricamente irrestricto). De hecho, hasta que en el siglo XVIII Fourier desarrollara sus series, nadie estaba en condiciones de asegurar que era empíricamente irrestricto, aunque sí, evidentemente, que era empíricamente irrefutable. Pero también es cierto que, hasta ese momento, tampoco nadie podía afirmar que fuera irrefutable simpliciter. Lo que prueba la serie de Fourier es que la ley fundamental de la TPP es empíricamente irrestricta y, por lo tanto, verdadera para toda aplicación intencional de la teoría. Evidentemente al probarlo, prueba también que no hay forma analítica de refutarlo y, por lo tanto, que no sólo era irrefutable empíricamente, sino también simpliciter. ${ }^{8}$

En el caso de la mecánica clásica, como ya hemos mencionado, la simplicidad de la ecuación que expresa la ley fundamental nos hace ver inmediatamente que, además de irrefutable, es empíricamente irrestricta porque se capta claramente cómo puede completarse trivialmente. Pero en el caso de la TPP, aun cuando la cuantificación existencial permite afirmar su irrefutabilidad empírica, la complejidad de su ecuación no permite, a simple vista, sostener que es empíricamente irrestricto ni que es irrefutable simpliciter. Que lo es, lo prueba Fourier. Por lo tanto, Fourier prueba que es empíricamente irrestricto y su estructura lógica nos garantiza que es empíricamente irrefutable. 
Y no sólo prueba que es empíricamente irrestricto, sino que provee de un método para encontrar el sed dada una determinada función; esto es, nos provee de un método para extender un modelo potencial hasta convertirlo en un modelo. Un método que, entonces, garantiza el éxito absoluto de la teoría. Con ese método, hemos hallado, por ejemplo, el sed apropiado para una órbita cuadrada, para una órbita en forma de flecha e, incluso, para una órbita que reprodujera el contorno del rostro de Homero Simpson.

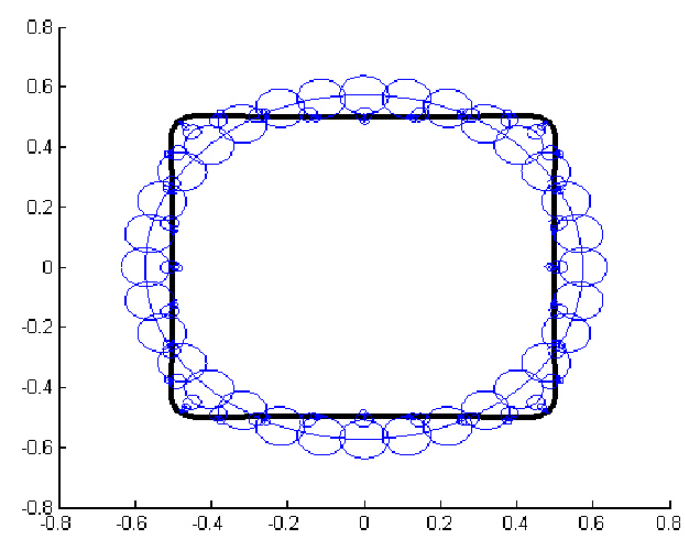

Aproximación a un cuadrado con 10 epiciclos

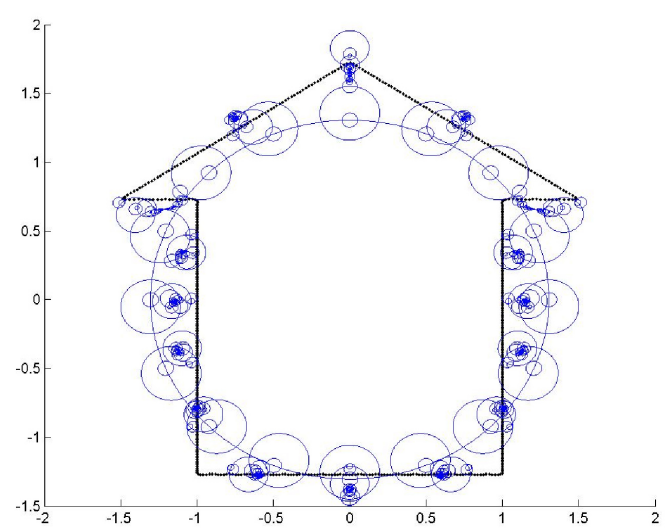

Aproximación a un cuadrado con 10 epiciclos

Principia 14(2): 211-239 (2010). 


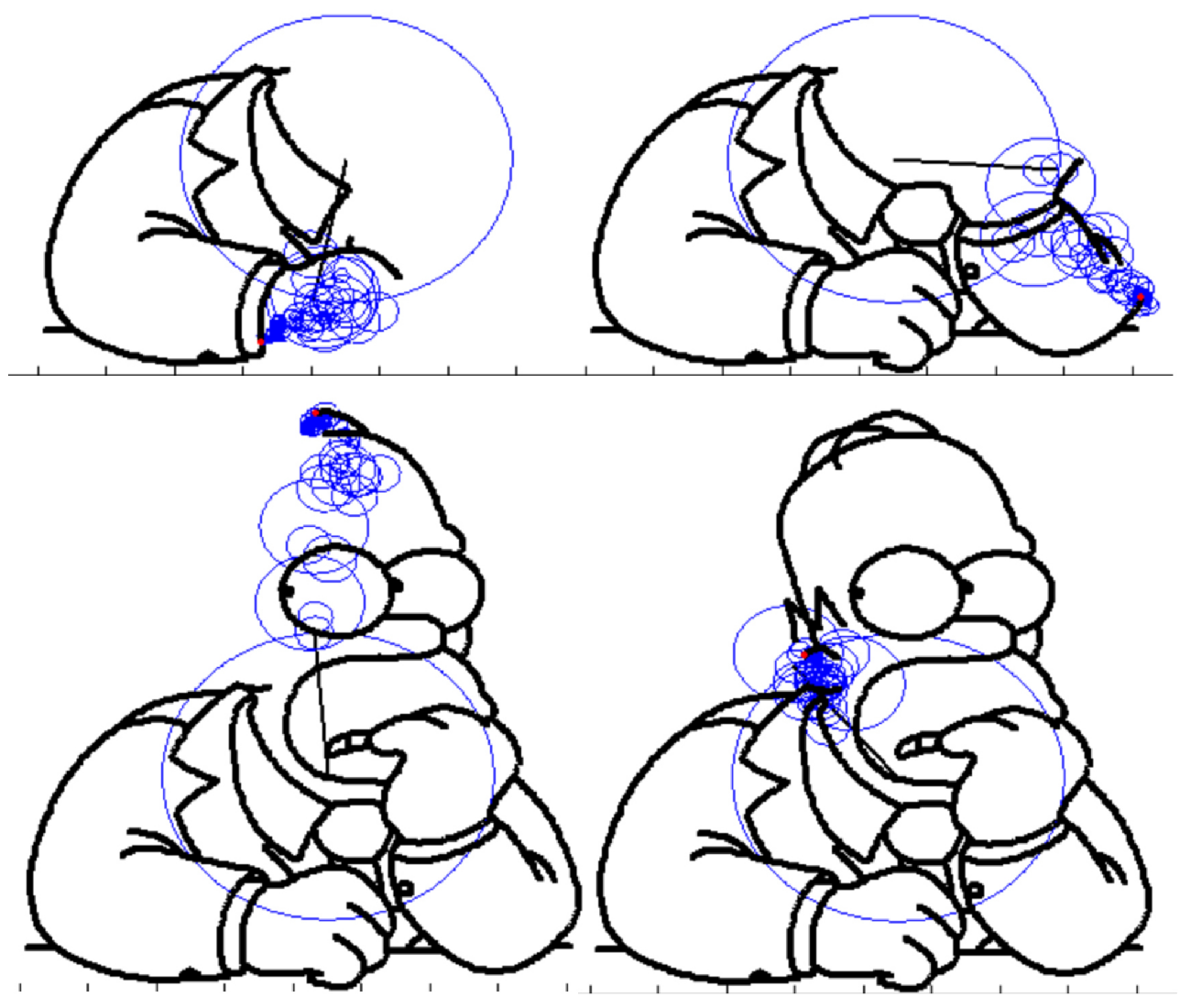

Aproximación a Homero Simpson con 10000 epiciclos

\section{Leyes especiales y condiciones de ligadura}

Como decíamos anteriormente, afirmar que la ley fundamental de una teoría es irrefutable no implica que la teoría lo sea, ya que la teoría es una realidad mucho más compleja. En general, las leyes fundamentales son restringidas por condiciones de ligadura, leyes especiales y vínculos interteóricos. ${ }^{9}$ En esta sección desarrollaremos, de la manera menos técnica posible, algunas leyes especiales de la TPP, relevantes para nuestro análisis. Para una mayor simplificación, nos centraremos exclusivamente en el cálculo de la longitud celeste, dejando de lado la latitud celeste. ${ }^{10}$

\subsection{Leyes que sirven para determinar los valores de las órbitas}

En primer lugar recordaremos la ley fundamental (LF) recortada a la longitud :

Principia 14(2): 211-239 (2010). 
(LF) En todo tiempo, la longitud calculada del punto móvil del sed debe coincidir con la longitud observada del planeta.

Pero no es ésta la única ley que se aplica a todos los modelos. Como hemos dicho, el deferente, la primera de las órbitas, da cuenta del movimiento medio del planeta, sobre el fondo de estrellas fijas. Por lo tanto:

(ley 1) el período de revoluciones del punto móvil del deferente (o sea del centro del primer epiciclo) debe coincidir con el período de revoluciones del planeta. O, también: la velocidad angular del deferente debe ser igual a la velocidad angular media del planeta. (Cfr. IX, 3; H2-214; T424.)

Estas dos, en principio, son las únicas leyes que se aplican a todos los modelos potenciales (a la órbita de cualquier planeta). Como se ve, la segunda impone una restricción sobre la primera, determinando la velocidad angular de la primera de las órbitas. $^{11}$

A estas dos leyes hay que agregar otras que se aplican sólo a aquellos planetas que presentan retrogradaciones en sus órbitas. Como el epiciclo es agregado al deferente con la intención de dar cuenta de las retrogradaciones, Ptolomeo exigirá lo siguiente:

(ley 2) el período de revoluciones del epiciclo debe coincidir con el tiempo comprendido entre dos retrogradaciones, es decir, el epiciclo dará una vuelta por cada retrogradación. (Cfr. IX, 3; H2-214; T424)

Como el sistema ptolemaico de hecho utiliza sólo un deferente y un epiciclo, ${ }^{12}$ las leyes 1 y 2 ya le permiten a Ptolomeo establecer la velocidad angular de todas las órbitas implicadas; pero, para poder calcular a partir de su sistema la longitud, no es suficiente con las velocidades, tiene que poder ubicar, en algún momento, los puntos que giran sobre las órbitas -el centro del epiciclo, esto es, el punto móvil del deferente y el punto móvil del epiciclo-; es decir, además de la velocidad, necesita alguna posición inicial. Ptolomeo sólo puede observar la longitud real del planeta, pero no puede "observar" la longitud de los puntos móviles. Sin embargo, su sistema es tal que:

(ley 3) en el medio de una retrogradación que se da sobre la línea absidal —la recta sobre la que se encuentran el centro de la Tierra y el punto excéntrico-, la longitud del planeta es igual a la longitud del punto móvil del deferente (o sea, del centro del epiciclo) y del punto móvil del epiciclo. ${ }^{13}$

Esta ley le permite ubicar las posiciones iniciales de los puntos móviles de las órbitas, ya que, en ese instante, coinciden con la del planeta que es observable. Lo único que queda por determinar es cómo calcular la ecuante, el punto excéntrico del deferente y los radios de las órbitas. Para ello no es necesario introducir leyes

Principia 14(2): 211-239 (2010). 
especiales, ya que los resultados se pueden obtener de la simple aplicación de la geometría euclidiana. En realidad, Ptolomeo no necesita calcular los valores absolutos de los radios de las órbitas, sino sólo la proporción entre ellos ya que, con radios proporcionales, los resultados son siempre iguales. ${ }^{14}$

Como se ve, entonces, a partir de tres leyes (además de la fundamental) y algunos cálculos, Ptolomeo es capaz de determinar todas las variables de su sistema: las velocidades angulares y una posición inicial de cada órbita, la proporción entre los radios y la ubicación del punto excéntrico y la ecuante. Con todos los valores determinados, calcular la longitud del punto móvil de todo el sistema es sólo una cuestión trigonométrica que Ptolomeo simplifica ofreciendo tablas (cfr. XI, 9-10; H2 426-428; T544-545).

\subsection{Análisis de la irrestricción empírica de las leyes que sirven para determinar los valores de las órbitas}

Como se recordará, para probar la irrestricción empírica de la ley fundamental, mostramos que la ley fundamental de la TPP podría entenderse como un desarrollo en series de Fourier y, así entendido, el mismo Fourier probaba que siempre se hallaría una solución. Enumeramos en su momento algunas restricciones que Fourier imponía a sus series y que Ptolomeo no tenía por qué respetar. Señalamos también que esas restricciones en nada afectaban al resultado porque lo único que probaban era que Ptolomeo tenía más libertad que Fourier. Pero mencionamos que existían también algunas restricciones que Ptolomeo imponía a su sistema y que Fourier no tendría por qué respetar y, de ser así, Fourier no bastaría para probar la irrestricción empírica de la ley fundamental más las leyes especiales. Es, por otro lado, el resultado que uno esperaría. Si realmente se trata de una teoría empírica, las leyes especiales deberían cortar con la irrestricción impidiendo que todos los modelos potenciales puedan extenderse a modelos actuales. Sin embargo, en lo que sigue probaremos que, incluso con estas restricciones, es posible convertir en modelo cualquier modelo potencial, es decir, sigue siendo una teoría empíricamente irrestricta.

Las leyes especiales que hemos visto determinan de una manera unívoca los valores de las dos primeras órbitas con criterios ajenos a los de las series de Fourier. Como hemos dicho, la velocidad angular de la segunda órbita es el doble del de la primera (su período es la mitad) y, en el caso de los radios, estaban determinados por la ecuación (10) que de ninguna manera garantiza que la proporción entre ellos será la que establecen los cálculos de Ptolomeo. La única restricción que sí es respetada automáticamente por Fourier es la ley 1 si identificamos al deferente con el término fundamental, ya que en sus series, el período del término fundamental coincide con el período de repetición de la función. ${ }^{15}$

Pero puede probarse que, dada una trayectoria cualquiera y un conjunto arbi-

Principia 14(2): 211-239 (2010). 
trario pero finito de órbitas circulares y uniformes con sus valores determinados, es posible encontrar un sed para esa trayectoria que incluya en él a todas las órbitas arbitrariamente definidas. Nótese que esto es más de lo que piden las leyes especiales, que sólo exigen que suceda en un par de órbitas. Sin embargo, para que la prueba sea relevante, debe concederse que Ptolomeo estaría dispuesto a agregar los epiciclos que fueran necesarios para ajustar la órbita en el caso de que los dos primeros no fueran suficientes. No parece estar lejos del espíritu de Ptolomeo ya que, en caso de la Luna y Mercurio, no titubeó en agregar otra pequeña órbita sobre la que giraba el punto excéntrico del deferente. ${ }^{16}$ Otro detalle que hay que tener en cuenta es que, en un sed, el orden de las órbitas no afecta el resultado final. Como en el fondo se trata de una suma vectorial, pueden colocarse las órbitas en el orden que se desee, sin que por ello se vea alterado el resultado. Por supuesto, nos estamos refiriendo sólo a la longitud del planeta, que es lo único que aquí estamos tratando.

La idea intuitiva de la prueba es muy sencilla. Teniendo una trayectoria determinada y un conjunto arbitrario de órbitas definidas, si restamos a la trayectoria del planeta la trayectoria resultante del conjunto arbitrario de órbitas nos dará una nueva trayectoria a la que, naturalmente, puede aplicársele Fourier. El resultado será un sed que describa esa nueva trayectoria que resultó de la resta a la trayectoria original de la trayectoria producida por las órbitas definidas arbitrariamente. Evidentemente, si a esa serie de Fourier le agregamos las órbitas definidas arbitrariamente obtendremos un conjunto de órbitas encadenadas que, por un lado, contengan las que queríamos incluir y, por otro, represente la trayectoria original que queríamos describir. He aquí la prueba formal.

1. Dada una trayectoria $\left(a_{t}\right)$, existe un conjunto de radios y frecuencias $(A)$ que describe la trayectoria, obtenida por desarrollo en series de Fourier.

$$
a(t)=\sum_{n=-\infty}^{n=+\infty} C_{a_{n}} e^{i \cdot n \cdot \omega_{a_{0}} \cdot t}
$$

2. Ahora bien, llamemos $D$ a un conjunto arbitrario de radios y frecuencias (órbitas) que queremos que sea el que esté incluido en el sed de la trayectoria $\left(a_{t}\right)$.

3. Luego, llamemos $c(t)$ a una segunda trayectoria imaginaria que coincide con $a_{t}$ menos el conjunto de órbitas $D$. Entonces el conjunto de radios y frecuencias de $c(t)$, que llamaremos $C$, es igual a $A-D$.

$$
c(t)=a(t)-\sum_{n=1}^{n=N_{0}} D_{n} e^{i \cdot \omega_{n} \cdot t}
$$

Principia 14(2): 211-239 (2010). 
4. Un simple pasaje muestra que $a_{t}$ es igual a $c(t)$ más el conjunto de órbitas $D$.

$$
a(t)=c(t)+\sum_{n=1}^{n=N_{0}} D_{n} e^{i \cdot \omega_{n} \cdot t}
$$

5. Pero si esto es así, es claro que un sed que contenga la serie de Fourier de $c(t)$ más el conjunto de órbitas $D$ —que es el conjunto de órbitas que queríamos incluir en el sed- será igual a $a_{t}$.

$$
a(t)=\sum_{n=-\infty}^{n=+\infty} C_{c_{n}} e^{i \cdot \omega_{c_{n}} \cdot t}+\sum_{n=1}^{n=N_{0}} D_{n} e^{i \cdot \omega_{n} \cdot t}
$$

6. Por lo tanto, queda probado que es posible construir un sed para una determinada órbita $a_{t}$ que contenga un número finito y arbitrario de órbitas $\mathrm{D}$. Y además, hemos provisto de un método para lograrlo.

Pero ¿son éstas las únicas restricciones que Ptolomeo impone a su sistema?

\subsection{Restricciones que expresan relaciones heliocéntricas desde un punto de vista geocéntrico}

La respuesta a la pregunta anterior parecería ser negativa. Dentro de los planetas que retrogradaban, era fácil distinguir dos grupos en función de cómo se comportaban con relación al Sol: aquellos cuya elongación —es decir, su distancia angular respecto del Sol - era limitada, o sea, siempre estarían a menos de una distancia angular $\eta$ y aquellos que podía encontrárselos a cualquier elongación. A los primeros los llamaremos planetas interiores y a los segundos, exteriores (cfr. IX, 1; H2-207; T419-420). Es importante aclarar que se trata aquí de una clasificación basada en criterios estrictamente no teóricos para TPP — es decir, no suponen la aplicación de la ley fundamental- y, además, en este caso particular, observacional. Existen, en Ptolomeo, algunas restricciones para los planetas de un tipo y para los de otro.

En el caso de los planetas interiores, cuya elongación —como hemos dicho- es limitada, Ptolomeo sostiene que

(condición 1) el período de revolución del deferente del planeta es igual al período de revolución medio del Sol (cfr. IX, 3; H2-218; T425). ${ }^{17}$

Si bien se trata de una nueva restricción, que un planeta interior que cumple con la ley 1 cumplirá también con esta condición (teniendo en cuenta, además, que el Sol cumple con la ley 1), es un enunciado analítico. En efecto, si un planeta tiene elongación limitada, quiere decir que nunca logra superar al Sol por más de una

Principia 14(2): 211-239 (2010). 
vuelta -ya que para pasarlo por una vuelta debería recorrer todas las elongaciones-. Si es así, el período de revolución del planeta y del Sol es el mismo y, aplicando a ambos la ley 1, se sigue que, por lo tanto, el período de revolución del deferente de ambos debe coincidir. No está de más enfatizar que lo analítico no es la condición 1 sino la proposición condicional que afirma que los planetas caracterizados TPP-no teóricamente como inferiores, si cumplen con la ley 1 y si el Sol también cumple con ley 1 , cumplirán con la condición 1.

En cambio, la relación es más compleja en el caso de los planetas exteriores. En ellos, sostiene Ptolomeo, la relación es la siguiente:

(condición 2) el período de revolución del epiciclo más el período de revolución del deferente de un planeta es igual al período de revolución del deferente del Sol (cfr. IX, 3; H2-214; T424).

Esta ley pretende dar cuenta de una regularidad empírica ya conocida desde los babilónicos: si contamos las vueltas que ha dado el planeta y le sumamos las retrogradaciones que se han producido en ese tiempo, el resultado coincidirá con las vueltas que ha dado el Sol. Sin embargo, la proposición condicional que afirma que si un planeta exterior y el Sol cumplen con la ley 1, el planeta cumplirá también con la condición 2, a diferencia del caso anterior, no es necesaria. No se sigue de que el planeta tenga elongación ilimitada más las leyes ya establecidas. Ahora bien, como el período de revolución del Sol y el del planeta quedan determinados unívocamente por la ley 1 y el período de revolución del epiciclo queda determinado unívocamente por la ley 2, no existe artilugio matemático que nos permita hacer cumplir esa ley si, de hecho, los valores no coinciden. Aquí sí se trata, por primera vez, de una restricción legítima, en el sentido de que excluye del conjunto de los modelos actuales a una serie de modelos potenciales, a saber todos aquellos en los que el período de revolución del planeta más el período de repetición de retrogradaciones no sea igual al período de revolución del Sol. Un planeta, por ejemplo, que tuviera la misma velocidad media de Marte pero que retrogradara dos veces por cada vez que lo hace de hecho Marte, es un modelo potencial que, en par con el del Sol, no respeta esta condición.

Es importante notar que si, como hemos señalado, las aplicaciones pretendidas de la condición 1 consisten en aquellos planetas retrogradantes que tienen elongación limitada y las de la condición 2 en aquellos retrogradantes que tienen elongación ilimitada, el conjunto de los planetas que retrogradan queda partido en dos subconjuntos por lo que, cualquier planeta que retrograde deberá caer bajo alguna de esas líneas de especialización, no es posible elaborar una tercera; en este caso tertium non datur. Así, frente a un modelo potencial que consistiera en un planeta con retrogradaciones, Ptolomeo no podría elaborar una nueva línea de especialización, 
pues, si tiene elongación limitada debería respetar (y respetará de hecho) la condición 1 y, si la elongación es ilimitada y Ptolomeo no estuviera dispuesto a modificar las aplicaciones pretendidas, debería respetar la condición 2.

Es probable, por supuesto, que de haberse encontrado Ptolomeo con un planeta que retrogradara y que, sin embargo, no respetara la condición 2, hubiera negado que la condición 2 se aplicara a todos los planetas con retrogradaciones y elongaciones ilimitadas, pero ello simplemente implica que su teoría, tomada en conjunto, tal cual él la presentó, habría sido refutada y, en el mejor de los casos, habría hecho alguna modificación no sustancial, reemplazando alguna ley especial. Por supuesto, todas las otras leyes especiales - las que sirven para la determinación de los valores - seguirían en pie, lo cual implica una diferencia jerárquica de las leyes, pero no que no fuera una restricción de la propuesta ptolemaica. De todas maneras, cualquier análisis contrafáctico debe ser tomado con pinzas porque la realmente escasa cantidad de aplicaciones propuestas de la teoría (apenas 7 planetas) hace muy difícil cualquier generalización. Pero la importancia que otorgaba a la condición 2 puede ser ilustrada con este detalle: para calcular la velocidad angular del deferente, en vez de calcular el período de revoluciones del planeta (aplicando la ley 1), directamente resta a la cantidad de retrogradaciones, las vueltas que ha dado el Sol, es decir, aplica la condición 2 (cfr. IX, 3; H2-218; T425).

Es importante notar que ésta, hasta ahora la única restricción que sería en principio refutable del sistema planetario ptolemaico aún sin modificar sus aplicaciones pretendidas, no ha sido nunca refutada y si consideramos al mundo como siendo heliocéntrico no podría serlo con planetas pertenecientes al sistema solar, ya que es verdad que si el planeta gira alrededor del Sol, esa relación, vista desde la Tierra, se respeta necesariamente. La ventaja de Copérnico, en todo caso, fue la de integrar esa relación aparentemente casual entre las leyes y mostrar su necesidad.

Pero no son éstas las únicas relaciones que la teoría ptolemaica establecía entre el movimiento de los planetas que retrogradaban y el del Sol. En efecto, ya desde antiguo se sabía que en el medio de una retrogradación, un planeta interior se encontraba más o menos en la misma línea del Sol, es decir, con elongación cercana a $0^{\circ}$, mientras que un planeta exterior se encuentra en oposición al Sol, es decir, con una elongación cercana a $180^{\circ} \cdot{ }^{18}$ Así, podríamos establecer las dos condiciones:

(condición 3) en el instante medio de una retrogradación, la elongación de un planeta interior es aproximadamente $0^{\circ}$.

(condición 4) en el instante medio de una retrogradación, la elongación de un planeta exterior es aproximadamente $180^{\circ} .19$

Aquí tampoco es posible ningún artilugio matemático ya que, como en el caso anterior, la relación que se está estableciendo es, en última instancia, reductible a lo empírico: la posición de los planetas y los instantes de su retrogradación no pueden 
manipularse. Y, al igual que en el caso de la condición 2, se trata de leyes que, de hecho, nunca han sido refutadas y no pueden serlo en planetas que giren alrededor del Sol porque, nuevamente, establece relaciones heliocéntricas observadas desde un sistema de referencia geocéntrico.

Debe quedar bien claro que estas tres condiciones (la condición 2, 3 y 4) vuelven a la TPP empíricamente restricta en combinación con las leyes especiales desarrolladas en la otra sección (ley 1, 2 y 3). El sólo hecho de, por ejemplo, exigir que la suma del período de revolución de las dos primeras órbitas sea igual al período de revolución del Sol (condición 2) no impone ninguna restricción real si no fuera porque la ley 1 determina el período de revolución de los deferentes y la ley 2 el del epiciclo. $^{20}$

\subsection{Condiciones de ligadura relacionadas con la distancia de los planetas}

Hasta ahora, todas las leyes especiales y condiciones genuinamente restrictivas expresan desde un punto de vista geocéntrico una propiedad que los planetas tienen por pertenecer a un sistema heliocéntrico. Sin embargo, hay al menos una forma de restringir los modelos potenciales extensibles a modelos que, si bien también está relacionada con el Sol, no supone el heliocentrismo. Esta forma de restringir los modelos potenciales implica asumir algunas tesis de la parte física de la astronomía ptolemaica, sin duda fuertemente inspirada en Aristóteles. A saber: que no existe el vacío, que la naturaleza no hace nada en vano y la concepción de que los epiciclos y deferentes eran esferas de existencia física.

La forma más sencilla de calcular la distancia de un planeta respecto de la Tierra es midiendo su paralaje. Pero, en la época de Ptolomeo, este método sólo era posible desde el punto de vista práctico para el caso de la Luna que, por encontrarse relativamente cerca de la Tierra, posee una paralaje lo suficientemente significativa como para ser medida. Ninguno de los otros planetas, incluyendo al Sol, presentan una paralaje observable. En una obra posterior al Almagesto, Las Hipótesis Planetarias, Ptolomeo intenta establecer las distancias de los planetas con un método sumamente ingenioso. Con su sistema de epiciclos y deferentes había sido capaz — como hemos visto- de establecer la proporción entre el radio del deferente y el del epiciclo y la proporción entre éstos y la excéntrica. En general, la distancia máxima que puede alcanzar un planeta coincide con la suma de los tres valores y la mínima con el resultado de restar al valor del deferente la suma de los otros dos. Así, es posible obtener resultados para la distancia máxima y mínima, pero siempre relativos a un deferente. Por ejemplo, en el caso de Venus, si consideramos que el deferente vale $60^{P}$ (partes), el radio del epiciclo es $43 ; 10^{P}$ y el de la excéntrica $1 ; 15^{P}$, la distancia máxima que puede alcanzar es $\left(60^{P}+43 ; 10^{P}+1 ; 15^{P}\right)=104 ; 25^{P}$ y la mínima $\left(60^{P}-43 ; 10^{P}\right.$ - 
$\left.1 ; 15^{P}\right)=15 ; 35^{P}$. La proporción entre ambas distancias, que expresa el grosor de la órbita es, entonces, ${ }^{104 ; 25} / 15 ; 35$. De la misma manera, Ptolomeo es capaz de calcular la distancia máxima y mínima de cualquier planeta, pero, evidentemente, estas distancias son relativas a cada deferente. Es como utilizar como unidad para medir la altura de cada persona la longitud del pie de esa persona: nos dará información, pero no nos permitirá saber quién es más alto que quién.

Ptolomeo, por otra parte, supone que no existe el vacío y que la naturaleza no hace nada en vano y, por lo tanto, afirma que la distancia máxima de un planeta tiene que coincidir con la mínima del planeta inmediato superior. Por lo tanto, si poseyera una distancia absoluta, podría calcular, en base a estas proporciones que expresan el grosor de las órbitas, la distancia máxima, media y mínima absoluta de cada planeta. Ahora bien, como hemos dicho, Ptolomeo había podido calcular la distancia máxima absoluta de la Luna. Ella debía coincidir, por lo tanto, con la distancia mínima de Mercurio; la distancia máxima de Mercurio se obtendría multiplicando la mínima ya obtenida por la proporción entre la máxima y la mínima. A su vez, la máxima de Mercurio coincidiría con la mínima de Venus y así sucesivamente hasta llegar a la distancia de la esfera de las estrellas fijas que debía coincidir con la distancia máxima de Saturno. ${ }^{21}$

Pero lo relevante es que estas nuevas relaciones no imponen ninguna restricción al sistema ptolemaico ya que las distancias absolutas no juegan ningún papel en el cálculo de las longitudes de los planetas, para el que es suficiente con las relativas. Si la Luna estuviera más lejos, todas las distancias serían más grandes; si estuviera más cerca, serían más pequeñas, pero en nada afectaría a las predicciones. ${ }^{22} \mathrm{Si}$ existiera, en cambio, alguna otra forma de calcular la distancia absoluta de algún planeta, entonces sí tendríamos una restricción empírica, ya que los valores para ese planeta, calculados mediante dos métodos distintos, deberían coincidir. Afortunadamente, existe tal método para el caso del Sol.

En el Almagesto, Ptolomeo había podido calcular, como hemos dicho, la distancia absoluta de la Luna midiendo su paralaje. Con ese dato y otros que obtiene de dos eclipses lunares introducidos en un ingenioso gráfico que representa un eclipse lunar $\mathrm{y}$ otro solar, encuentra para la distancia entre la Tierra y el Sol un valor de $1210^{r t}$ (radios terrestres). En este cálculo intervienen, técnicamente, sólo tres datos: la distancia absoluta de la Luna, el radio aparente de la Luna y el Sol (que coinciden) cuando ésta se encuentra a su máxima distancia y el radio aparente de la sombra de la Tierra proyectada sobre la Luna cuando, nuevamente, ésta se encuentra a su máxima distancia. Como se ve, aquí no interviene ningún valor de los sistemas de los planetas. Tenemos, por lo tanto, dos cálculos independientes que deben coincidir en un mismo valor y estamos, entonces, frente a una restricción empírica.

El valor de la distancia media del Sol, obtenida desde el primer cálculo, compromete el grosor de las órbitas de Mercurio y Venus — los únicos que Ptolomeo 
considera más cercanos que el Sol-y, por lo tanto, son los modelos potenciales de estos dos planetas - y de cualquier otro planeta interior, si lo hubiera - los que quedan restringidos por esta condición unida al cálculo independiente de la distancia Tierra-Sol. Los valores absolutos de los radios, de las órbitas y de la excéntrica de los planetas interiores tienen que ser tales que el límite máximo de sus órbitas encastradas coincida con la distancia mínima del Sol, obtenida mediante el cálculo de los eclipses. Evidentemente, una alteración de esos valores con el objetivo de hacerlo coincidir con el valor de la distancia del Sol comprometería el éxito de la predicción de las longitudes. Recuérdese que sólo restringe a los planetas interiores.

Se trata, por lo tanto, de una genuina restricción empírica que no es fruto del carácter heliocéntrico de los planetas, muy al contrario, sólo tiene sentido plantearla en el sistema ptolemaico. Siendo el sistema ptolemaico falso, es de esperar que los valores no coincidan. Sin embargo, coincidían, y de una manera asombrosa. Partiendo de los datos obtenidos en el Almagesto, Ptolomeo redondea la distancia máxima de la Luna en $64^{r t}$. Coloca, por lo tanto, la posición mínima de Mercurio a esa distancia y, tomando como la proporción entre las distancias máximas y mínimas de Mercurio valor de $88 / 34$, calcula la distancia máxima de Mercurio $166^{\text {rt }}$ que coincide, obviamente, con la distancia mínima del siguiente planeta, Venus. La proporción entre las distancias de Venus es $104 / 16$, por lo que su distancia máxima será $1079^{r t}$. Ahora bien, la distancia media del Sol es de $1210^{r t}$, y la diferencia entre la distancia media y la mínima es de ${ }^{1} / 24$ parte de la distancia media del Sol, por lo que la mínima será de $1160^{r t}$. La diferencia es de apenas $81^{r t}$, menos del $7 \%$. Una coincidencia más que sorprendente. Pero en realidad es mucho más sorprendente si tenemos en cuenta que, si Ptolomeo no hubiera redondeado los valores obtenidos en el Almagesto y hubiera sido más cuidadoso con sus cálculos, la distancia máxima de Venus alcanzaría $1189^{r t}$ que se pasaría de la mínima del Sol por apenas $29^{r t}$, sólo el $2,4 \%$, una coincidencia maravillosa teniendo en cuenta las dificultades de observación y cálculo que podía tener Ptolomeo. Pero puede aún ser más asombrosa si tenemos en cuenta este último detalle. En las Hipótesis Planetarias, Ptolomeo corrige brevemente los parámetros de Mercurio. Si se realizan los cálculos con los parámetros corregidos, se obtiene para la distancia máxima de Venus un valor de aproximadamente $1146^{r t}$, con apenas $14^{r t}$ de diferencia, lo que representa un $1,1 \%$. Simplemente increíble.

Esta restricción tiene una diferencia epistémica respecto de las otras. Si bien todas son genuinas, las anteriores eran, de alguna manera, a posteriori: observada esa relación en los planetas, se la establece como ley, mientras que esta última es una consecuencia necesaria de la teoría interpretada físicamente.

\section{Conclusión}

A partir del análisis que hace Moulines de los principios guías, hemos profundizado en la irrestricción empírica del sistema de epiciclos y deferentes propuesto por PtoPrincipia 14(2): 211-239 (2010). 
lomeo. Hemos mostrado que hay que distinguir entre la irrefutabilidad empírica, la irrefutabilidad simpliciter y la irrestricción empírica y que mientras la primera dependía de su estructura lógica, las otras dos eran probadas aplicando al sed el análisis en series de Fourier. Luego analizamos una a una las principales leyes especiales de la teoría que, de ser genuinas, deberían cortar con esa irrestricción. Mostramos que todas aquellas que sirven para determinar los valores de las variables de las órbitas (velocidades angulares, posiciones iniciales, radios y excéntricas), incluso combinadas, no bastaban para excluir ni siquiera un modelo potencial. Pero no sucede lo mismo si agregamos las leyes que vinculan algunos de esos valores con la posición del Sol. En efecto, cuando, además de todo lo anterior, exigimos que las velocidades y las posiciones de los planetas mantengan una relación particular con la velocidad y posición del Sol, la teoría pierde su irrestricción empírica volviéndose, recién en esta instancia, una teoría genuinamente empírica ya que, en palabras de Popper (2002: 35), "prohíbe ciertas cosas". Curiosamente, todas estas restricciones no hacen más que expresar desde un sistema de referencia geocéntrico propiedades que tienen los planetas por pertenecer a un sistema heliocéntrico. Por lo tanto, podría decirse que la teoría de Ptolomeo, considerada sólo matemáticamente, sólo es refutable en la medida en que refleja propiedades heliocéntricas. No por hacerlo, pero de hecho es así. Por lo que las mismas restricciones que la vuelen refutable hacen que su sistema no pueda de hecho ser refutado porque son leyes "verdaderas". Si aquí terminara el análisis, se vería por qué el ataque al sistema planetario de Ptolomeo por parte de los heliocentristas, no podía provenir de un intento de refutación de su teoría planetaria en sí, puesto que eran empíricamente equivalentes. ${ }^{23} \mathrm{Y}$ aumentaría la plausibilidad de la hipótesis histórica que afirma que fue atacada principalmente a causa de su matrimonio indisoluble con la cosmología aristotélica. Pero, como hemos mostrado, existe una restricción relacionada con el cálculo de la distancia de los planetas que expresa una propiedad, en cambio, que le pertenece al sistema geocéntrico en cuanto tal siempre que sea interpretado físicamente, es decir, que se acepte que los epiciclos y deferentes son esferas reales. Sin embargo, es una restricción empírica que sólo restringe a los modelos potenciales de planetas interiores. Desde una posición heliocéntrica —antes de la utilización del telescopio que aportaría nuevas complicaciones- debería haber sido éste el dato atacado por Copérnico, pues era el único que realmente lo diferenciaba de un sistema heliocéntrico. Sin embargo, curiosamente, Copérnico no sólo no criticó el resultado del cálculo de la distancia de la Tierra al Sol de Ptolomeo sino que, incluso, modificó sus propios valores con el fin de ajustarlo al valor obtenido por Ptolomeo, como prueba J. Henderson (1973) mostrando las tachaduras y correcciones de los distintos borradores del De Revolutionibus.

Creemos que el análisis aquí presentado aporta claridad respecto de la refutabilidad de la teoría ptolemaica, confirmando una creencia habitual, esto es, que gran 
parte de la caída del sistema ptolemaico se debe a su estrecha relación con una física aristotélica. En efecto, hemos visto que, si se consideran aisladamente los modelos ptolemaicos de cada planeta, cada uno de ellos es irrefutable (ley fundamental más las tres leyes especiales). Si, en cambio, se los pone en relación con el Sol, se vuelve refutable pero no refutada porque las restricciones son empíricamente verdaderas (condiciones 1 a 4). Si, finalmente, se la interpreta como un sistema físico (aristotélico), la teoría se vuelve refutable y refutada a partir del cálculo de la distancia de la Tierra al Sol, aunque de hecho, históricamente, esa refutación nunca se la haya explicitado. ${ }^{24}$

\section{Bibliografía}

Aaboe, A. 1960. The Insignificance of Fourier Expansions for the Understanding of Ptolemy's Models. Isis 51: 565.

Balzer, W., Moulines, C. U. and Sneed, J. 1987. An Architectonic for Science. The Structuralist Program. Dordrecht: Reidel.

Carman, C. C. 2011. El Elemento teórico básico del Sistema de Epiciclos y Deferentes de Ptolomeo, Agora. en prensa.

Díez, J. A. y Moulines, C. U. 1999. Fundamentos de Filosofía de la Ciencia. 2da. edición. Barcelona: Ariel.

Díez, J. A. y Lorenzano, P. 2002. La concepción estructuralista en el contexto de la filosofía de la ciencia del siglo XX. En Díez, J.A. y P. Lorenzano (eds.) Desarrollos actuales de la metateoría estructuralista: problemas y discusiones. Quilmes: Universidad Nacional de Quilmes/Universidad Autónoma de Zacatecas/Universidad Rovira i Virgili, pp. 13-78.

Evans, J. 1998. The History and Practice of Ancient Astronomy. Oxford: Oxford University Press.

Goldstein, B. R. 1967. The Arabic version of Ptolemy's Planetary Hypotheses. Transactions of the American Philosophical Society, New Series, 57(4): 1-55.

Halma, M. 1813-1816. Composition Mathématique de Claude Ptolémée. 2 vols. Paris: Chez Henri Grand.

Hanson, R. 1960. The Mathematical Power of Epicyclical Astronomy. Isis 51: 150-8.

-. 1961. The Insignificance of Aaboe's Remarks for Understanding Hanson's Article. Isis 52: 98.

Heiberg, J. L. (ed.) 1898-1903. Claudii Ptolemaei Opera quae exstant omnia. Vol. I, Syntaxis Mathematica, 2 vols. Leipzig: Teubner.

- (ed.) 1907. Claudii Ptolemaei Opera quae exstant omnia. Vol. II, Opera Astronomica Minora. Leipzig: Teubner.

Henderson, J. 1973. On the Distances between Sun, Moon, and Earth according to Ptolemy, Copernicus, and Reinhold. Ph.D. diss., Yale University.

Gingerich, O. 1975. Crisis Versus Aesthetic in the Copernican Revolution. Vistas in Astronomy 17: 85-94.

Griffiths, R. 1988. Was There a Crisis before the Copernican Revolution? A Reappraisal of Gingerich's Criticisms of Kuhn. PSA: Proceedings of the Biennial Meeting of the Philosophy of Science Association Vol. 1: 127-32

Principia 14(2): 211-239 (2010). 
Kuhn, Th. [1957] 1993. La revolución copernicana. Traducción de Domènec Bergadà de la versión original publicada en inglés titulada The Copernican Revolution. Planetary Astronomy in the Development of Western Thought (Harvard University Press, Cambridge). Buenos Aires: Planeta.

Lorenzano, P. 2006. Fundamental Laws and Laws of Biology. En Ernst, G. y K.-G. Niebergall (eds.) Philosophie der Wissenschaft - Wissenschaft der Philosophie. Festschrift für C.Ulises Moulines zum 60. Geburstag. Paderborn: Mentis-Verlag, pp. 129-55.

Manitius, K, 1912-1913. Ptolemäus, Handbuch der Astronomie. Deutsche Übersetzung von K. Manitius. 2 vols. Leipzig: Teubner.

Moulines, C. U. [1978] 1982. Forma y función de los principios-guía en las teorías físicas. En Exploraciones metacientíficas. Madrid: Alianza, pp. 88-107. (Primera publicación como "Cuantificadores Existenciales y Principios-Guía en las Teorías Físicas", Crítica 10: 5988, 1978.)

Neugebahuer, O. 1969. The Exact Sciences in Antiquity, New York: Dover.

- . [1957] 1969 The Exact Sciences in Antiquity. Second edition. New York: Dover.

- 1975. A History of Ancient Mathematical Astronomy. Studies in the History of Mathematics and Physical Sciences 1. 3 vols. Berlin: Springer.

Pedersen, O. 1974. A Survey of the Almagest. Acta Historica Scientirarum Naturalium et medicinalium. Vol. 30. Odense: Odense University Press.

Pedersen, O. y Jones, A. 2010. A Survey of the Almagest: With Annotation and New Commentary by Alexander Jones. New York: Springer.

Pérez Sedeño, E. 1987. Las Hipótesis de los Planetas. Introducción y notas de E. Pérez Sedeño. Traducciones de J. G. Blanco y A. Cano Ledesma. Madrid: Alianza.

Popper, K. 2002 Búsqueda sin término: una autobiografía intelectual. Madrid: Alianza.

Taliaferro, R. 1952. The Almagest by Ptolemy. En Great Books of the Western World, Chicago (Encyclopaedia Britannica), Vol. 16.

Toomer, G. J. 1998. Ptolemy's Almagest. (Primera edición: London: Durkworth, 1984) Princeton: Princeton University Press.

Woodruff, A. E. 1961. Notes and Correspondence. Isis 52(1): 93-4.

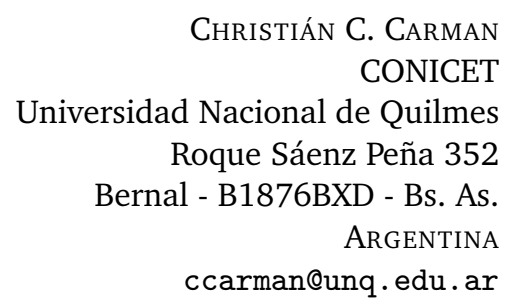

Resumo. Afirmar que a teoria planetária da Antiguidade, tal qual a propôs Ptolomeu, era — ao menos até a aparição do telescópio — uma teoria irrefutável, é um lugar comu. O objetivo deste trabalho é analisar em que sentido o sistema de epiciclos e deferentes proposto por Ptolomeu para dar conta do movimento planetário é irrefutável e em que sentido o é. Para tanto, utilizaremos o marco conceitual da concepção estruturalista e, em particular, a análise que faz Moulines dos 'princípios guia'.

Palavras-chave: Refutabilidade, astronomia antiga, Ptolomeu, princípios guia.

Principia 14(2): 211-239 (2010). 


\section{Notas}

${ }^{1}$ La introducción más sencilla al estructuralismo, pero que cuida la precisión, puede encontrarse en Díez y Moulines (1999): 327-366. Otra introducción precisa, accesible y breve puede verse en Díez y Lorenzano (2002): 55-71. Para un estudio, todavía introductorio, pero mucho más detallado puede consultarse Balzer, Moulines y Sneed (1987).

${ }^{2}$ La traducción al castellano del texto griego es mía. Los textos del Almagesto serán citados según el uso habitual, indicando primero el libro y capítulo de la Syntaxis y luego el tomo y la página de la edición crítica de Heiberg (H2-208) a lo que agregaré, para mayor comodidad, la página de la traducción de Toomer (T 420). La edición griega clásica, entonces, es Heiberg (1898-1903), existiendo dos traducciones al inglés, de las cuales la segunda es infinitamente mejor: Taliaferro (1952) y Toomer (1988), una al francés: Halma (18131816) y una al alemán: Manitius (1912-1913). Como obras introductorias al Almagesto sin duda la mejor (aunque no sin errores) es Pedersen (1974), felizmente ha salido una nueva edición corregida y comentada por Jones (Pedersen y Jones 2010); también puede verse Neugebauer (1975), de mayor amplitud. Una introducción didáctica y seria a la astronomía antigua puede encontrarse en Evans (1998). La otra obra de Ptolomeo que citaremos aquí es Las Hipótesis Planetarias. En Heiberg (1907) hay parte de la obra, el resto, con el original árabe y traducido al inglés en Goldstein (1967). Existe traducción castellana de toda la obra (aunque con errores) en Pérez Sedeño (1987).

${ }^{3}$ En realidad son tres, pues hay que incluir el conjunto de órbitas sobre las que gira la excéntrica, en el caso que gire (como en el sistema de epiciclos y deferentes de la Luna y de Mercurio). Estas órbitas no son en sentido estricto ni deferentes ni epiciclos, pero este detalle es irrelevante para los objetivos del presente trabajo.

${ }^{4}$ Aquí "inversamente proporcional" debe ser leído sólo cualitativamente: es decir, a un incremento de brillo corresponde una disminución de la distancia y viceversa, ya que al brillo no se le asignaba ningún valor numérico.

${ }^{5}$ Una reconstrucción formal de la teoría puede verse en Carman (2011).

${ }^{6}$ Aquí "limitaciones" debe entenderse de una manera particular. No son nuevos requisitos que se agregan a las series de Fourier, sino que son las propiedades que Fourier encontró que era necesario respetar para construir una serie de forma tal que tienda a la función deseada cuando el número de los términos tiende a infinito. Es como sostener que si alguien desea obtener un número positivo como resultado del producto de otros dos números, debe "limitarse" a utilizar pares de números con el mismo signo. Como luego veremos, algunas de las leyes especiales de Ptolomeo son "limitaciones" en el mismo sentido: imponían requisitos que debían seguirse para que pudiera cumplirse con la ley fundamental. Lo relevante es que estas limitaciones son distintas a las que Ptolomeo establece.

${ }^{7}$ Aaboe (1960) critica a Hanson afirmando que, puesto que el sistema de Ptolomeo utilizaba puntos ecuantes y, por lo tanto, el movimiento sobre el deferente no es uniforme medido desde su centro, no puede ser correctamente representado por una serie de Fourier para el cual siempre el movimiento es uniforme. Pedersen (1974): 35, n. 5 sostiene lo mismo. Hanson responde en (1960b). Sin embargo, no necesitamos afirmar que no hay diferencias entre el planteo de Fourier y el de Ptolomeo, sino sólo que el desarrollo en series de Fourier sirve para probar la irrestricción empírica de la ley fundamental de Ptolomeo. De todas maneras, Woodruff (1962) demuestra que, si bien es imposible reproducir exactamente el

Principia 14(2): 211-239 (2010). 
efecto que produce una ecuante con un número finito de epiciclos, agregando dos o a lo sumo cuatro epiciclos (dependiendo de la proporción entre la ecuante y el radio del deferente) puede suprimirse la ecuante sin diferencias observables para los límites de observación ptolemaicos.

${ }^{8}$ Aquí se ve la diferencia que señalábamos anteriormente entre los enunciados existenciales no matemáticos y los matemáticos. En los primeros, cuyo ejemplo era "existe un cuervo blanco", es posible probar que es irrefutable simpliciter sin probar que sea verdadero. En los segundos, en cambio, al menos no hemos encontrado una forma de probar que es imposible probar que sea falso (probar su irrefutabilidad simpliciter) sin probar al mismo tiempo que es verdadero (probar su irrestricción empírica).

${ }^{9}$ Para una discusión adicional sobre las leyes en la concepción estructuralista ver Lorenzano (2006), en especial, nota 11.

${ }^{10}$ La misma idealización hace Ptolomeo al comienzo del tratamiento de los planetas, aunque luego, por supuesto, desarrolla la teoría de las latitudes en el libro XIII. Cfr. IX, 6; H2-254; T443-444.

${ }^{11}$ En sentido estricto, el deferente no tiene por qué ser la primera de las órbitas - y no lo es en el caso de la Luna y de Mercurio-, pero aquí esto no es relevante. Para una discusión más técnica, véase Carman (2011).

${ }^{12}$ Como hemos dicho, dejaremos de lado por cuestiones de simplicidad el tratamiento de la órbita excéntrica de la Luna (que de todas maneras no retrograda) y de Mercurio.

${ }^{13}$ Ver nota 19.

${ }^{14}$ En el caso de los planetas exteriores primero calcula la dirección y el valor proporcional del punto ecuante. Para ello elige tres observaciones en las que la otra anomalía (aquella de la que dará cuenta el epiciclo) sea inexistente. Ello se produce en las oposiciones y conjunciones con el Sol, ya que en esos casos el planeta y el centro del deferente están alineados con la Tierra. Elije oposiciones porque, evidentemente, los planetas no son observables en las conjunciones. Una vez calculada la dirección y el valor del punto ecuante, prueba que ése no puede ser el punto sobre el que gira el deferente y sostiene (aunque sin probarlo) que el centro del deferente es un punto que, ubicado sobre la misma línea, está a mitad de camino entre la ecuante y la Tierra. Obtiene así el punto excéntrico. Con esos valores y una nueva observación, puede calcular el valor proporcional del radio del epiciclo. En el caso de los planetas interiores, en cambio, calcula primero la línea absidal (aquella sobre la que estará la excéntrica y el ecuante) ya que funciona como eje de simetría de las máximas elongaciones. Y luego, ya obtenido este valor, obtiene mediante un cálculo muy sencillo la distancia de la excéntrica (que sí puede obtenerla, en este caso, a partir de datos empíricos) y el valor del radio del epiciclo. Luego, con otro cálculo sencillo, obtiene la ecuante (ver X, 6; H2-317; T480, donde marca las diferencias entre el procedimiento para planetas interiores y para exteriores).

${ }^{15}$ Que las frecuencias de los términos de la serie de Fourier sean múltiplos de una fundamental, implica que cada término realiza un número entero $(2,3,4$, etc.) de ciclos por cada uno del fundamental. Para que la función se repita en un dado instante, todos los términos deben tomar en ese momento el valor que tenían inicialmente. Dado que el primer término es el que tiene la menor frecuencia, también es el que tarda más en repetirse y cuando esto ocurre, todos los otros se han repetido exactamente un número entero de veces y tienen su valor inicial. Por lo tanto, el período de repetición de la función, es el período del término

Principia 14(2): 211-239 (2010). 
fundamental.

16 También podría apoyarse la plausibilidad histórica señalando que, como es una creencia común sin duda popularizada por Kuhn [1957] 1993, el desarrollo del paradigma ptolemaico consistió en ir agregando cada vez más epiciclos menores. Sin embargo, hay suficiente evidencia histórica para cuestionar seriamente esta tesis. Cfr. Gingerich (1975) y también Griffith (1998) quien argumenta a favor de Kuhn.

${ }^{17}$ Esta restricción y las que siguen no son, en sentido estricto estructuralista, leyes especiales sino condiciones de ligadura porque no restringen al elemento teórico básico sino a pares de elementos teóricos (en este caso, al par Sol-planeta interior). Por ello las llamaremos condiciones en vez de leyes. De todas maneras, esta cuestión técnica no es determinante para el desarrollo de nuestra tesis central.

${ }^{18}$ La relación es más precisa, pero aclararlo implica nuevas complicaciones por lo que dejaremos en el cuerpo del texto la versión simplificada de la ley y explicaremos en esta nota los detalles. En realidad, la relación no se debe medir con la elongación del Sol, sino del Sol medio. El Sol medio es un término teórico elaborado por Ptolomeo que representa el punto en el que se encontraría el Sol si tuviera un movimiento uniforme. Ptolomeo eligió para el Sol un sistema con una sola órbita, pero excéntrica. Podría haber elegido un sistema con un deferente con centro en el Sol y un epiciclo — de hecho él analiza ambas posibilidades-, si hubiera elegido el sistema de epiciclo y deferente, el Sol medio representaría el centro del epiciclo. Por otro lado la elongación con el Sol medio es $0^{\circ}$ en el caso de los planetas interiores y $180^{\circ}$ en el de los exteriores sólo cuando el centro de la retrogradación se produce sobre la línea absidal, es decir, en la misma línea de la que hablaba la ley 3. Mientras la ley 3 ubicaba en línea recta al planeta, el centro y el punto móvil del epiciclo, estas condiciones agregan que el Sol está alineado también con ellos en ese instante, en el caso de los planetas interiores del mismo lado, y, en el de los exteriores, del otro lado de la Tierra pero siempre sobre la misma recta.

${ }^{19}$ Cfr. X, 6; H2 319-320; T483. En este texto se expresa la ley 3 combinada ya con la condición 4, es decir coloca bajo la misma línea al planeta (y por lo tanto el punto móvil del epiciclo) al centro del epiciclo, a la excéntrica, a la Tierra y al Sol medio más la del Sol relacionada y mencionado, incluso, lo de las paralelas, para los planetas exteriores. Y agrega lo que podría ser un teorema derivado de la combinación de las leyes 1, 2 y 3 y de la condición 4 ya que si en un momento están alienadas y las velocidades son constantes, la línea que une el centro del epiciclo con el centro del deferente (el punto excéntrico) es siempre paralela a la línea que une a la Tierra con el Sol medio.

${ }^{20}$ Probablemente también deba considerarse a la relación que existe entre la distancia del punto excéntrico y la del punto ecuante respecto de la Tierra (la distancia del punto ecuante mide el doble que la del punto excéntrico) y que estén alineadas como una ley especial de la teoría (IX, 5; H2-252; T443). Esta ley valdría para todos los retrogradantes (excepto para Mercurio), pero es muy discutido cómo realmente Ptolomeo obtuvo esa relación (cfr. Pedersen 1974: 277-8 y 306 y la bibliografía allí citada).

${ }^{21}$ Esta relación entre las distancias máximas y mínimas de los planetas no es, técnicamente, una ley especial sino una condición de ligadura, ya que vincula modelos entre sí, estableciendo relaciones entre ellos.

${ }^{22}$ En realidad, el alejamiento de la Luna no es conflictivo, pero sí puede serlo el acercamiento, ya que, si los planetas están demasiado cerca de la Tierra, la paralaje sería perceptible y

Principia 14(2): 211-239 (2010). 
no tenerla en cuenta afectaría las predicciones de longitud. Ptolomeo es consciente de ello (IX, 1; H2-207; T419-420). De todas maneras, en Las Hipótesis Planetarias parece olvidarlo, porque Mercurio a su mínima distancia estaría a la máxima de la Luna y a esa distancia la paralaje es perceptible.

${ }^{23}$ Dejando de lado, por supuesto, el caso de las fases de Venus que eran inobservables sin telescopio.

${ }^{24}$ Agradezco a Pablo Lorenzano y a todo el grupo de investigación que él dirige, a Jose A. Díez y a Hernán Grecco. Y muy especialmente a Ramiro Serra con el que trabajamos juntos en una versión anterior de este trabajo. Este trabajo fue realizado con la ayuda del proyecto de investigación PICT 2010-0319 de la Agencia Nacional de Promoción Científica y Tecnológica.

Principia 14(2): 211-239 (2010). 